\title{
Stephan's Quintet: Implications for Compact Groups
}

\author{
Jack W. Sulentic \\ Department of Physics and Astronomy, University of Alabama, \\ Tuscaloosa, AL, USA 35487
}

\begin{abstract}
We review old and new observational data for Stephan's Quintet (SQ), the most famous compact galaxy group. Recently obtained multi-wavelength data has helped to clarify the past and present evolutionary history of this group. If SQ is typical of the compact group phenomenon, then: 1) the groups evolve slowly, resisting merging, due to the injection of energy from high velocity intruders and 2) show low levels of enhanced star formation because individual component ISM's are quickly stripped via intruder collisions with resultant gas either too hot or cold/diffuse to form many stars. Infall of residual near nuclear gas may also stimulate the development of AGN.
\end{abstract}

\section{Introduction}

Stephan's Quintet is perhaps the best studied example of the compact group phenomenon. As number 92 in the Hickson (1982) catalog (HCG), it satisfied fairly rigorous selection criteria involving richness $(N=5$ galaxies), isolation and compactness $\left(\mu_{G}=22.3\right.$ within $\left.r=1.6 \mathrm{arcmin}\right)$. SQ is, in fact, one of the most compact and high surface brightness groups in the HCG sample with or without its discordant redshift member (Sulentic 1993; Prandoni et al. 1994). This is consistent with the idea that it is one of the more evolved groups if it is typical of the phenomenon and if most compact groups have a similar origin. Compact groups have received a great deal of observational and theoretical attention over the past 15 years. We propose to address some of the questions that are raised by their existence as physically dense systems. We supplement published observational results with unpublished data obtained with ISO/Calar Alto (with C. Xu and R. Tuffs), ROSAT (with G. Trinchieri and W. Pietsch) and San Pedro Martir (Fabry-Perot imaging with D. Dultzin-Hacyan and M. Rosado). We also address some of the apparent paradoxes that the recent studies have revealed. In each case we answer the questions, and attempt to resolve the paradoxes, using $\mathrm{SQ}$ as an assumed prototype of the compact group class.

\section{Questions About Compact Groups}

\subsection{A Discordant Redshift Excess?}

Many compact groups (43/100 in HCG; Sulentic 1997) contain one or more discordant redshift members $\left(\Delta V \geq 5000 \mathrm{~km} \mathrm{~s}^{-1}\right)$. How could so many groups 
be involved if the discordant galaxies are chance projections? Part of the answer lies in the fact that the HCG selection criteria were particularly effective in finding such discordant configurations. If one corrects the HCG for: a) groups that fail the original selection criteria and b) groups that were "created" by the discordant members (Sulentic 1997) one is left with 61 groups with at least 4 accordant redshift members. SQ is one of the 6 groups in this refined sample with an internal discordant member (a discordant galaxy that falls inside the accordant group diameter circle). Is the discordant galaxy (NGC7320) a physical member? Two independent observational results suggest not. 1) Redshifts for galaxies within a few degrees of SQ show values that are similar to either: a) the accordant group $\left(\sim 6500 \mathrm{~km} \mathrm{~s}^{-1}\right)$ or b) the discordant $\left(\sim 800 \mathrm{~km} \mathrm{~s}^{-1}\right)$ galaxy (Lynds 1972; Shostak et al. 1984). This is consistent with the hypothesis that we are observing two "sheets" of galaxies along the same line of sight with respective mean distances of $\left(\mathrm{H}_{0}=75 \mathrm{~km} \mathrm{~s}^{-1} \mathrm{Mpc}^{-1}\right) \sim 11$ and $87 \mathrm{Mpc}$. 2) Recent $\mathrm{H} \alpha$ (Moles et al. 1997 and new Calar Alto data) and HI (Verdes-Montenegro private communication) images of NGC7320 show no sign of dynamical perturbation; in stark contrast to the accordant members of SQ.

\subsection{How Do Compact Groups Form?}

At least four models have been advanced to explain compact groups. Not all of them have dealt explicitly with compact group formation. 1) The lack of observational evidence for the predicted rapid dynamical collapse of dense groups motivated some to argue that they are accordant chance projections rather than physically dense aggregates (Mamon 1986; Hernquist et al. 1995). All four accordant members of SQ show evidence for interaction and dynamical evolution. The weakest evidence, for NGC7317, involves its unambiguous connection to the rest of the group via a diffuse optical halo (Moles et al. 1998).

2) Those less troubled by the lack of evidence for mergers argue that compact groups form continuously by the collapse of loose groups (Barnes 1989; Ramella et al. 1994; Diaferio et al. 1994). SQ is certainly associated with a larger distribution of galaxies which have, and are, playing a role in its evolution. However it shows no evidence for merger activity and the large number of expected SQ-like merger postcursors in SQ-like environments have not been found (Sulentic and Rabaca 1994). A recent attempt to refine the alignment model would use X-ray data to distinguish between real and false groups (Ostriker et al. 1995). This assumes that real groups often have associated diffuse X-ray emission. ROSAT HRI mapping (Pietsch et al. 1997) shows that SQ is dominated by X-ray emission that does not arise in a diffuse halo. New unpublished HRI data shows that the X-ray emission is dominated by a shock feature and the nucleus of NGC7319. The numerous H I clouds scattered between the galaxies (Shostak et al. 1984) also argue against a hot diffuse intergalactic medium in this group (see also Williams et al. in this proceedings for similar conclusion concerning HCG16).

3) An attempt to model both the formation and evolution of the groups (Governato et al. 1996) views them as long lived systems that grow by secondary infall onto primordial merger nucleation sites. The problem with this approach is that most compact groups do not contain a first-ranked elliptical that might represent a nucleation site. SQ contains two elliptical members but neither is 
luminous enough to be a candidate visible-matter nucleation site. 4) Recent models invoking massive non-cuspy dark matter haloes (Athanassoula et al. 1997) appear to produce very long lived groups. A conservative dynamical age for SQ exceeds 1 Gyr so it has already outlived the predictions of class 2) models.

\subsection{How Do Compact Groups Evolve?}

SQ appears to be evolving through a sequence of encounters with high velocity intruders. There is a stable core of three galaxies (NGC 7317, 18a, 19) with essentially zero line of sight velocity dispersion and embedded in the aforementioned luminous optical halo.

In the past this core has interacted with NGC7320c a fainter galaxy located less than one group diameter $\mathrm{NE}$. The (unfortunately uncertain) velocity difference is $\Delta \mathrm{V} \approx 600 \mathrm{~km} \mathrm{~s}^{-1}$ relative to the core. Twin tidal tails (see e.g Arp 1973) extend from NGC7319, and from behind NGC7320 in the direction of NGC7320c, suggesting that it has passed though the group more than once. The former tail is narrower and higher surface brightness suggesting that it is the more recent signature of an encounter. A large amount of $\mathrm{H} I$ with velocities very similar to the stripped spiral NGC7319 is superposed on the region of this tail (Shostak et al. 1984; Williams et al. in preparation) consistent with the hypothesis that NGC7320c removed it during the most recent passage. New ISO, Calar Alto ( $\mathrm{H} \alpha)$ and San Pedro Martir ( $\mathrm{H} \alpha$ Fabry-Perot) observations reveal one active emission region in this tail with a velocity consistent with the surrounding H I ( $\left.6600 \mathrm{~km} \mathrm{~s}^{-1}\right)$. Surface photometry of the tails (Schombert et al. $1990)$ indicate that they are very blue $(B-V \sim 0.2-0.4)$ consistent with an origin in the stripped disks of late-type galaxies and/or indicative of star formation that has now largely ceased. The morphology of NGC7320c (RSXS0 in RC3) is consistent with the hypothesis that it may be a stripped spiral (Moles et al. 1997) (note that a published $\mathrm{H}$ I redshift for this galaxy is spurious). The only gas that can now be unambiguously assigned to NGC7319 is involved with the Seyfert 2 nucleus and environs (Aoki et al. 1996). It is tempting to attribute the onset of AGN activity to the same source that stripped the ISM of NGC7319.

The present activity in SQ involves an ongoing collision between NGC7318b and the previously stripped ISM of the group $\left(\Delta V \approx 1000 \mathrm{~km} \mathrm{~s}^{-1}\right)$. The strongest evidence for an ongoing collision involves the detection of a radio (van der Hulst and Rots 1981), X-ray (Pietsch et al. 1997) and $\mathrm{H} \alpha /[\mathrm{NII}]$ (Moles et al. 1997) "arc" between NGC7319 and 7318b. The most straightforward interpretation of this feature is that it represents a shock interface between NGC7318b and the SQ ISM. All of the arms/filaments surrounding NGC7318ab can be attributed to the intruder based on published (Plana et al. 1999) and unpublished $\mathrm{H} \alpha$ Fabry-Perot measures. H I in this region (Shostak et al. 1984; Williams et al., in preparation) also shows velocities consistent with the hypothesis that it is the unshocked remnant of the intruder ISM.

\subsection{Where is the Interaction-Starburst Effect?}

Studies of binary galaxies reveal evidence for a multiwavelength emission enhancement attributed to interaction induced star formation (see e.g. Kennicutt et al. 1987; Xu and Sulentic 1991). Similar and stronger enhancement is observed from the so-called merger systems. Paradoxically, compact groups show 
only weak evidence for a similar enhancement even though they represent the apparent extremum of the galaxy-galaxy interaction phenomenon (Moles et al. 1994; Sulentic and Rabaca 1994; Allam et al. 1996; Verdes-Montenegro et al. 1998) and are expected to be the site of frequent galaxy-galaxy mergers. Recent ISO MIR and FIR observations confirm this deficit for SQ. First, the new data show the benefits of higher resolution: much of the emission is found to be due to the late-type and discordant redshift NGC7320. The strongest source associated with accordant SQ is due to the Seyfert nucleus in NGC7319. It is clear from the above discussed $\mathrm{HI}$ and X-ray data that most of the gas in SQ is either too hot, cold and/or diffuse to show the level of star formation enhancement observed from the bound gas-rich disk of a dynamically perturbed spiral galaxy. The ISO data do reveal isolated sites of star formation. The most unusual involves a small starburst north of the current intruder NGC7318b (Xu et al. 1999). It occurs where one arm of the intruder appears to intersect the upper edge of the shock feature and yields a rate of $0.8 M_{\odot} \mathrm{yr}^{-1}$. The estimated age of the burst $\left(1-2 \times 10^{7}\right.$ years $)$ is consistent with the crossing time of the intruder and the cooling time for the X-ray shock feature - all indicative of an ongoing event.

\subsection{How Do Compact Groups Avoid Merging?}

SQ has clearly outlived the predictions for many models of compact group evolution. The model of Barnes (1989) implied total coalescence after $\sim 1.2 \mathrm{Gyr}$ with partial mergers beginning after a very few crossing times. The multifold forms of evidence for interaction discussed above (tidal tails, $\mathrm{H}$ I completely stripped) and dynamical evolution (diffuse optical halo, a multiplicity (NGC 7317, 18a and 20c) of early-type galaxies in a noncluster environment) in SQ suggest that $1 \mathrm{Gyr}$ is a conservative estimate for the age of this group. Yet the galaxies in SQ show no obvious signs of merging. The only evidence for inward directed activity involves the Seyfert nucleus. The other forms of activity are outward directed tending to diffuse the mass in the group. It is therefore reasonable to ascribe the longevity to the periodic arrival of neighboring galaxies that inject energy into the system. A restricted version of this scenario was proposed for compact groups by Governato et al. (1991). If the intruders generally show large velocity differences as observed in SQ (a more accurate redshift is needed for NGC7320c) then the mechanism must involve transfer of their excess kinetic energy to the group. The stages in the process can be well illustrated by a hierarchical series of virial calculations (Moles et al. 1997) where: 1) the group core (NGC7317, $18 \mathrm{a}$ and 19) is bound, 2) the addition of NGC7320c yields a marginally bound system and 3) the addition of the new intruder NGC7318b yields an unbound system. The twin tidal tails, if both are associated with NGC7320c, independently suggest that it has been captured by SQ. The velocities of the intruders in SQ are inconsistent with the typical velocity dispersions observed in loose groups. If the groups are to act as nucleation points, they require an order of magnitude more mass $\left(10^{13} M_{\odot}\right.$ rather than $\left.10^{12} M_{\odot}\right)$ than is observed, in order to accelerate neighbors (from rest) to infall velocities in the range 500-1000 $\mathrm{km} \mathrm{s}^{-1}$. Thus elements of the above mentioned formation models 2 (loose group infall), 3 (groups as "magnets") and 4 (large DM haloes) are required.

Do other HCG groups show evidence for high velocity intruder activity. Figure 1 shows a plot of magnitude and velocity difference for group mem- 


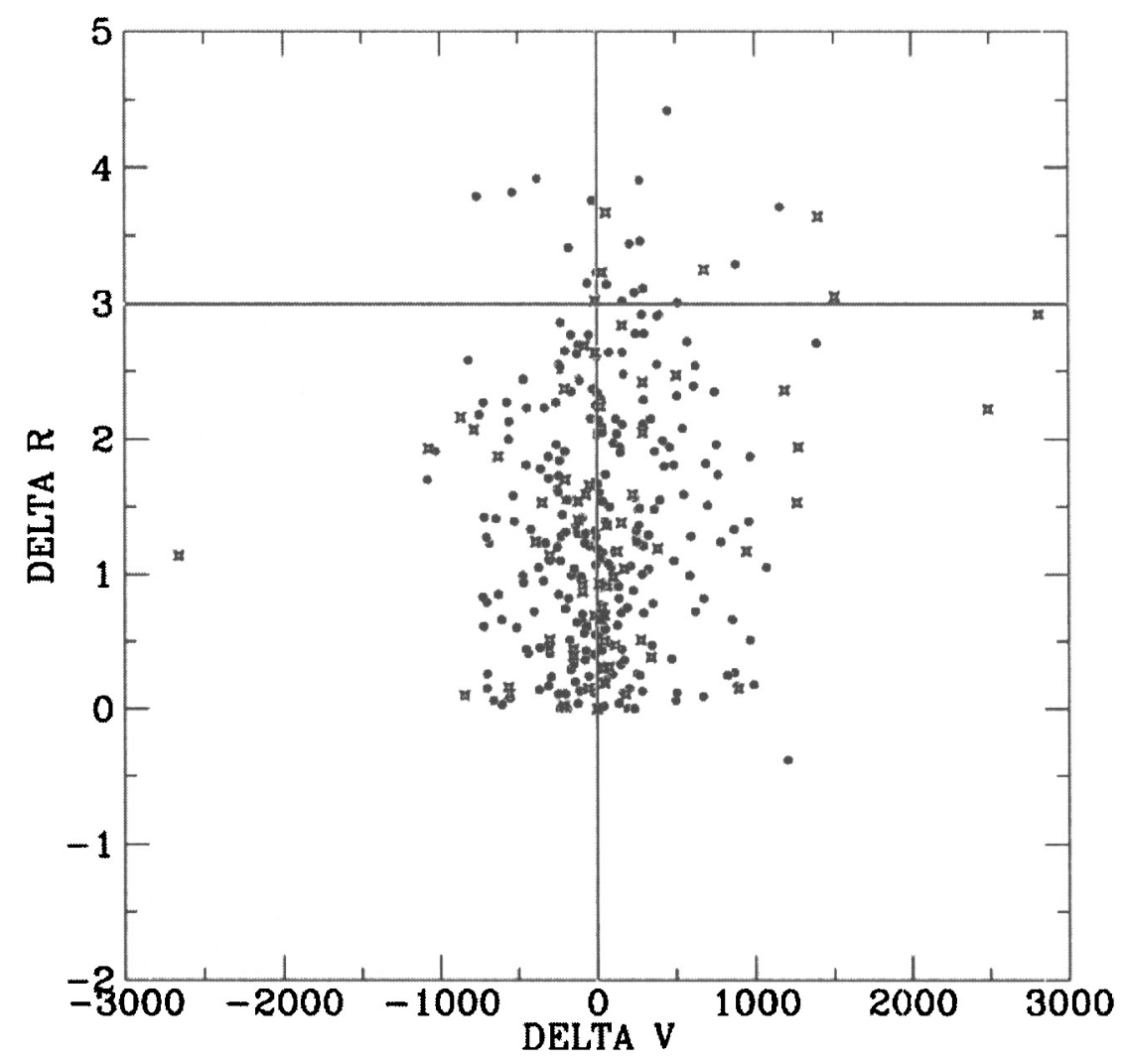

Figure 1. A differential Hubble diagram for compact group members. We plot the $\mathbf{R}$-band magnitude and recession velocity differences for all group galaxies relative to their respective first-ranked members. Differentials are in the sense faintest - brightest.

bers relative to their respective first-ranked member. Magnitude and velocity differences in this differential Hubble diagram are in the sense faintest minus brightest. Thus no galaxies with negative $\mathrm{R}$ band magnitude differences should be observed. Those with differences larger than 3.0 violate the magnitude concordance criterion used in creating the HCG. The velocity differentials show that intruders like NGC7318b are not unprecedented. The differential velocity distribution shows an envelope of, technically, HCG members although such large velociy differences indicate that these galaxies are not (yet?) bound to their respective groups. The distribution of velocity differences is distinctly nonGaussian in the wings due to the presence of the small excess of high velocity outliers.

Note that members of false groups (mostly triplets plus an outlier) are marked with stars in figure 1 . Their small numbers show a stronger concentra- 
tion of core velocities with larger velocity amplitude outliers. Are these groups in the process of formation by infall or dissolution by dynamical ejection of members? This apparent difference from stable groups requires confirmation with a larger triplet sample. If confirmed, the large relative velocities of these outliers would favor the dissolution hypothesis.

It is interesting to point out that the most famous and first-discovered compact group was included in the HCG only because of the discordant member. NGC7320 is the brightest member of the group but the four accordant group members show apparent magnitudes within $\Delta R=3.0$ allowing $\mathrm{SQ}$ to satisfy the HCG magnitude concordance criterion (because nearby NGC7320c is more than three magnitudes fainter than NGC7320). If NGC7320 were not projected on the group, the four accordant members would not lie sufficiently far from NGC7320c to satisfy the isolation criterion. If one tried to create a somewhat larger group including NGC7320c, other nearby galaxies with similar apparent magnitude would cause the new group to fail the isolation criterion. The message in this illustration is that the HCG probably does not contain all the parts of the compact group puzzle. Many of the groups with infalling members will not be included because they will be in mid-infall and will often link the core of the group to the loose group environment of which the galaxies are a part. Such groups would not be included in the HCG because they would not be sufficiently isolated. SQ is a rare case where an intruder (NGC7318b) is now entering the group and where the brightness of the projected discordant redshift member (NGC7320) prevents the older fainter intruder (NGC7320c) from linking it to its larger scale environment.

\section{References}

Allam, S., Assendorp, R., Longo, G., Braun, M. and Richter, G. 1996, A\&AS, $117,39 \mathrm{~A}$

Aoki, K., Ohtani, H., Yoshida, M. and Kosugi, G. 1996, AJ, 111, 140

Arp, H. 1973, ApJ, 183, 411

Athanassoula, E., Makino, J. and Bosma, A. 1997, MNRAS, 286, 825A

Barnes, J. 1989, Nature, 338, 123

Diaferio, A., Geller, M. and Ramella, M. 1994, AJ, 107, 868

Governato, F., Bhatia, R. and Chincarini, G. 1991, ApJ, 371, L15

Governato, F., Tozzi, P. and Cavaliere, A. 1996, ApJ, 458, 18

Hernquist, L., Katz, N. and Weinberg, D. 1995, ApJ, 442, 57

Hickson, P. 1982, ApJ, 255, 382

Kennicutt, R., Keel, W., van der Hulst, J., Hummel, E. and Roettiger, K. 1987, AJ, 93, 1011

Lynds, R. 1972, in External Galaxies and Quasi-Stellar Objects, ed. D. S. Evans, (Reidel: Dordrecht), p. 376

Mamon, G. A. 1986, ApJ, 307, 426

Moles, M., del Olmo, A., Perea, J., Masegosa, J., Marquez, I. and Costa, V. 1994, A\&A, 285, 404 
Moles, M., Sulentic, J. W. and Marquez, I. 1997, ApJ, 485, L69

Moles, M., Marquez, I. and Sulentic, J. W. 1998, A\&A, 334, 473

Ostriker, J., Lubin, L. and Hernquist, L. 1995, ApJ, 444, L61

Pietsch, W., Trinchieri, G., Arp, H. and Sulentic, J. W. 1997, A\&A, 322, 89

Plana, H., Mendes de Oliveira, C., Amram, P., Bolte, M., Balkowski, C. and Boulesteix, J. 1999, ApJ, 516, L69

Prandoni, I., Iovino, A. and Mac Gillivray, H. T. 1994, AJ, 107, 1235

Ramella, M., Diaferio, A., Geller, M. and Huchra, J. 1994, AJ, 107, 1623

Schombert, J., Wallin, J. and Struck-Marcell, C. 1990, AJ, 99, 497

Shostak, S., Sullivan, W. T. III, and Allen, R. 1984, A\&A, 139, 15

Sulentic, J. W., 1993, in Progress in New Cosmologies, eds. H. Arp, C. R. Keys and K. Rudnicki, (Plenum: New York), p. 49

Sulentic, J. W. 1997, ApJ, 482, 640

Sulentic, J. W. and Rabaça, C. 1994,ApJ, 429, 531

Van der Hulst, J. M. and Rots, A. H. 1981, AJ, 86, 1775

Verdes-Montenegro, L., Yun, M., Perea, J., Del Olmo, A. and Ho, P. 1998, ApJ, 497,89

Xu, C. and Sulentic, J. W. 1991, ApJ, 374, 407

Xu, C., Sulentic, J. W., Tuffs, R. 1999, ApJ, 512, 178 